\title{
TNM-PNI: a novel prognostic scoring system for patients with gastric cancer and curative D2 resection
}

This article was published in the following Dove Press journal: Cancer Management and Research

\author{
Zi-Ming Gao' \\ Rui-Ying Wang ${ }^{2}$ \\ Peng Deng' \\ Peng Ding' \\ Chen Zheng' \\ Bin Hou' \\ Kai Li' \\ 'Department of Surgical Oncology \\ and General Surgery, The First \\ Affiliated Hospital of China Medical \\ University, Shenyang I I000I, China; \\ ${ }^{2}$ Department of Ultrasound, The First \\ Affiliated Hospital of China Medical \\ University, Shenyang II000I, China
}

Correspondence: Kai Li

Department of Surgical Oncology and General Surgery, The First Affiliated Hospital of China Medical University, I55 Nanjing North Street, Heping District, Shenyang II000I, China

Email cmu_likai@hotmail.com
Purpose: Gastric cancer (GC) is one of the most common malignancies and has a high mortality rate. In recent years, several nutritional or inflammatory biomarkers have been shown to effectively predict the prognosis of tumors. In this study, we intended to establish a prognostic scoring system for GC patients.

Patients and methods: Our study included a total of 501 GC patients who were diagnosed with GC stage I-III and received curative gastrectomy with D2 lymphadenectomy between January 2011 and December 2012. Survival analysis was performed using Kaplan-Meier and log-rank tests. Two Cox multivariate models, one for continuous and one for categorical variables, were established to identify independent prognostic factors. All statistical analyses were performed using SPSS 20.0.

Results: Univariate and multivariate analyses revealed that tumor-node-metastasis (TNM) stage, preoperative prognostic nutritional index (PNI), and adjuvant therapy were independent prognostic factors for GC patients. We established a new composite variable, TNM-PNI, which was confirmed to be a major prognostic factor for curative D2 resection, independent of whether adjuvant therapy was administered. GC patients with higher TNM-PNI scores always had worse cancer outcomes. In addition, we found that adjuvant therapy might be beneficial for the survival of GC patients with TNM-PNI $=4$ or 5 .

Conclusion: Preoperative PNI plays a distinctly subsidiary role to the TNM stage when predicting patient prognosis. TNM-PNI is a novel and an effective prognostic index for GC patients with curative D2 resection and a good supplement for the National Comprehensive Cancer Network Guidelines.

Keywords: tumor-node-metastasis stage, prognostic nutritional index, adjuvant therapy, cancer outcome prognosis

\section{Introduction}

Gastric cancer (GC) is the fourth most common malignancy and the second leading cause of cancer-related death worldwide. ${ }^{1} \mathrm{GC}$ patients from China account for $\sim 40 \%$ of the total global incidence. Surgery with adjuvant therapy is the preferred intervention for GC, and gastrectomy with D2 lymphadenectomy has become the standard procedure. This intervention is especially common in East Asia because of the high incidence rate of GC. Despite surgical intervention, the 5-year survival rate of GC patients is $<40 \%$, even after curative resection. Therefore, identifying accurate prognostic factors and an effective scoring system to predict the survival time of GC patients is imperative for improving the treatment of GC. 
In recent years, nutritional and inflammatory status have been recognized as factors for predicting cancer outcomes. ${ }^{2}$ Several studies have reported on preoperative biomarkers and composite indexes, such as albumin, ${ }^{3}$ platelet count, body mass index (BMI), Glasgow Prognostic Score (GPS), ${ }^{4,5}$ neutrophil-lymphocyte ratio (NLR), ${ }^{6-8}$ and prognostic nutritional index (PNI). ${ }^{9-11}$ While all these biomarkers are easily measured, noninvasive, and effective, it is still controversial which of them represents the best predictor of GC prognosis.

In this retrospective study, we intended to investigate independent prognostic factors of GC and to establish a novel prognostic scoring system for patients who are treated with curative D2 resection. The results of this study are expected to improve the prediction of GC prognosis and guide the development of postoperative treatment strategies for surgeons.

\section{Patients and methods Patient selection}

We retrospectively analyzed the clinicopathologic and follow-up data of 501 patients who were diagnosed with GC and received curative D2 resection at the First Affiliated Hospital of China Medical University from January 2011 to December 2012. Gastrectomy with D2 lymphadenectomy was performed according to the guidelines of the Japanese Research Society for Gastric Cancer. The inclusion criteria of patients were as follows: 1) GC was confirmed as stage I-III by histopathology, 2) curative D2 resection was performed without neoadjuvant chemo-radiotherapy, 3) postoperative adjuvant therapy regimens included chemotherapy or targeted therapy, and 4) detailed clinicopathologic and follow-up data were obtained for every patient. Furthermore, among the 501 patients, 268 stage II-III patients with adjuvant therapy and another 192 stage I-III patients without adjuvant therapy were selected to identify the prognostic value of the novel scoring system of the National Comprehensive Cancer Network (NCCN) guidelines for GC. This research was approved by the Ethics Committee of China Medical University, and written informed consent for this study was obtained from each patient.

\section{Data collection}

We collected the following data for each patient: age, gender, tumor size (longest diameter), radical degree, histological type, tumor-node-metastasis (TNM) stage, albumin level, platelet count, fibrinogen level, NLR, platelet-lymphocyte ratio (PLR), PNI, adjuvant therapy, and OS time. Routine laboratory analysis of blood was performed 7 days preoperatively. Cutoff values for biomarkers were determined by their Youden index of the receiver operating characteristic curve and the results of previous studies. Follow-up was completed by December 2017. TNM stages were classified according to the $7^{\text {th }}$ edition of the American Joint Committee on Cancer (AJCC) classification system.

\section{Definition of NLR, PLR, and PNI}

NLR was calculated as neutrophil count $\left(\right.$ per $\left.\mathrm{mm}^{3}\right) / \mathrm{lym}$ phocyte count (per $\mathrm{mm}^{3}$ ), PLR was calculated as platelet count (per $\mathrm{mm}^{3}$ )/lymphocyte count (per $\mathrm{mm}^{3}$ ), and PNI was calculated as 10 -fold albumin concentration $(\mathrm{g} / \mathrm{dL})$ plus 0.005 -times total lymphocyte count (per $\mathrm{mm}^{3}$ ). For subsequent calculations, patients with $\mathrm{PNI} \leq 49$ were assigned PNI $=1$ and patients with PNI $>49$ were assigned PNI $=2$.

\section{Definition of TNM-PNI}

A novel prognosis scoring system, "TNM-PNI," was established combining TNM and PNI, both of which were found to be independent prognostic factors in our analysis. We assigned a score of 1-3 to the relative TNM stage (I-III) and a score of 1-2 to PNI (1-2). Finally, based on the results of the B value and hazard ratio (HR) value in Model 2 of the Cox analysis, we calculated the TNM-PNI score for each patient according to the formula: TNM-PNI $=2 \times \mathrm{TNM}$ score $-1 \times$ PNI score.

\section{Statistical analysis}

Continuous and categorical variables were expressed as mean \pm SD and frequency, respectively. To identify the independent prognostic factors, we performed univariate and multivariate analyses with the Cox proportional hazards model. Two models were established in the Cox analysis, one including the continuous variables and the other including the categorical variables. Correlation analyses between preoperative PNI and other characteristics were performed by independent sample $t$-tests and chi-square tests. In addition, Kaplan-Meier analysis and the log-rank test were used to evaluate the difference in prognosis between TNM-PNI groups. Statistical analysis was performed using SPSS 20.0, and $p<0.05$ was considered to be statistically significant.

\section{Results}

\section{Clinicopathologic characteristics}

We retrospectively included 501 patients with stage I-III GC in this study. Their baseline clinicopathologic characteristics are shown in Table 1. There were 364 male (72.7\%) and 137 
Table I General characteristics of 50 I GC patients with curative D2 resection

\begin{tabular}{|c|c|}
\hline Variables & No. of patients (\%) \\
\hline \multicolumn{2}{|l|}{ Age (years) } \\
\hline$\leq 60$ & $274(54.7)$ \\
\hline$>60$ & $227(45.3)$ \\
\hline \multicolumn{2}{|l|}{ Gender } \\
\hline Male & $364(72.7)$ \\
\hline Female & $137(27.3)$ \\
\hline \multicolumn{2}{|l|}{ Diameter (cm) } \\
\hline$\leq 4.0$ & $232(46.3)$ \\
\hline$>4.0$ & $269(53.7)$ \\
\hline \multicolumn{2}{|l|}{ Radical degree } \\
\hline$A$ & 360 (7I.9) \\
\hline B & $|4|(28.1)$ \\
\hline \multicolumn{2}{|l|}{ Histological type } \\
\hline Well differentiated & $112(22.4)$ \\
\hline Poorly differentiated & $255(50.9)$ \\
\hline Mixed & $134(26.7)$ \\
\hline \multicolumn{2}{|l|}{ TNM stage } \\
\hline I & II4 (22.8) \\
\hline II & $139(27.7)$ \\
\hline III & $248(49.5)$ \\
\hline \multicolumn{2}{|l|}{ Platelet $\times 10^{9} / \mathrm{L}$} \\
\hline$\leq 300$ & $414(82.6)$ \\
\hline$>300$ & $87(17.4)$ \\
\hline \multicolumn{2}{|l|}{ Albumin (g/dL) } \\
\hline$\leq 4.0$ & $136(27.1)$ \\
\hline$>4.0$ & $365(72.9)$ \\
\hline \multicolumn{2}{|l|}{ Fibrinogen (g/L) } \\
\hline$\leq 3.5$ & $242(48.3)$ \\
\hline$>3.5$ & $259(5 I .7)$ \\
\hline \multicolumn{2}{|l|}{ NLR } \\
\hline$\leq 3.0$ & $435(86.8)$ \\
\hline$>3.0$ & $66(13.2)$ \\
\hline \multicolumn{2}{|l|}{ PLR } \\
\hline$\leq 130$ & $283(56.5)$ \\
\hline$>130$ & $218(43.5)$ \\
\hline \multicolumn{2}{|l|}{ PNI } \\
\hline$\leq 49$ & $151(30.1)$ \\
\hline$>49$ & $350(69.9)$ \\
\hline \multicolumn{2}{|l|}{ Adjuvant therapy } \\
\hline No & $192(38.3)$ \\
\hline Yes & $309(61.7)$ \\
\hline
\end{tabular}

Abbreviations: GC, gastric cancer; TNM, tumor-node-metastasis; NLR, neutrophil-lymphocyte ratio; PLR, platelet-lymphocyte ratio; $\mathrm{PNI}$, prognostic nutritional index.

female $(27.3 \%)$ patients, and the mean age was 59.2 years. Of the 501 patients, $114(22.8 \%)$ had stage I, $139(27.7 \%)$ had stage II, and $248(49.5 \%)$ had stage III. The mean follow-up period was 43.1 months. Furthermore, the 1-, 3-, and 5-year OS rates of the patients were $85.0 \%, 62.1 \%$, and $53.9 \%$, respectively. Among the 501 patients, 309 received postoperative adjuvant therapy and $86.7 \%$ (268/309) were diagnosed with stage II-III.

\section{Results of the univariate and multivariate analysis}

The univariate analysis revealed that age, gender, tumor size, radical degree, TNM stage, albumin, fibrinogen, NLR, PLR, and PNI might be associated with the prognosis of GC patients $(p<0.05)$. Both multivariate models of the Cox analysis, using continuous and categorical variables, respectively, showed that TNM stage [Model 1: HR $(95 \% \mathrm{CI})$ $=2.965$ (2.378-3.698), $p=0.000$; Model 2: HR $(95 \%$ CI) $=2.957$ (2.369-3.690), $p=0.000$ ], PNI [Model 1: HR $(95 \%$ CI $=0.972(0.954-0.991), p=0.004 ;$ Model 2: HR $(95 \% \mathrm{CI})$ $=0.647(0.497-0.844), p=0.001]$, and adjuvant therapy [Model 1: HR $(95 \% \mathrm{CI})=0.569(0.434-0.747), p=0.000 ;$ Model 2: HR $(95 \% \mathrm{CI})=0.567(0.432-0.745), p=0.000]$ are the independent prognostic factors of the OS for patients with curative D2 resection (Table 2). Higher TNM stage or lower preoperative PNI was always associated with worse prognosis of GC. Moreover, adjuvant therapy, as another major prognostic factor, could improve the long-term OS of stage II-III, but not that of patients with stage I in our study (Figure 1A-C).

\section{Relationship between $\mathrm{PNI}$ and other characteristics}

The preoperative PNI level was associated with age, tumor size, TNM stage, albumin level, fibrinogen level, NLR, and PLR $(p<0.05)$ (Table S1). Notably, patients with a high PNI $(>49)$ were always relatively younger, had smaller sized tumors, and presented with an earlier TNM stage compared with patients in the low-PNI group. Furthermore, we found that PNI could effectively predict the prognosis of TNM stages, especially for GC stages I and II (Figure 2A-F).

\section{Prognostic value of TNM-PNI}

Relative to TNM stage, the TNM-PNI system showed improved accuracy in predicting prognosis (Figure $3 \mathrm{~A}$ and $\mathrm{B})$, and a higher TNM-PNI score always indicated worse OS of GC. Because the difference in prognoses did not reach statistical significance, we generalized TNM-PNI $=1$ and 2 as a single group (Figure 3C). By Cox multivariate analysis, TNM-PNI [Model 1 and Model 2: HR $(95 \% \mathrm{CI})=1.812$ (1.632-2.010), $p=0.000$ ] and adjuvant therapy [Model 1 and Model 2: $\mathrm{HR}(95 \% \mathrm{CI})=0.597(0.456-0.782), p=0.000]$ were demonstrated to be the major independent prognostic factor for our $501 \mathrm{GC}$ patients (Table S2).

\section{Role of TNM-PNI for NCCN guidelines}

According to the NCCN guidelines for GC, for the postoperative management of D2 gastrectomy, a total of 268 
Table 2 Univariate and multivariate survival analysis of OS in 50I GC patients with curative D2 resection

\begin{tabular}{|c|c|c|c|c|c|}
\hline \multirow[t]{2}{*}{ Variables } & \multicolumn{2}{|l|}{ Univariate analysis } & \multicolumn{3}{|c|}{ Multivariate analysis } \\
\hline & HR (95\% Cl) & $p$-value & B-value & HR (95\% Cl) & p-value \\
\hline \multicolumn{6}{|c|}{ Model I (Continuous variables) } \\
\hline Age & $1.015(1.003-1.028)$ & $0.018^{*}$ & & & \\
\hline Gender & $1.350(1.025-1.778)$ & $0.033^{*}$ & & & \\
\hline Diameter & $1.148(1.095-1.204)$ & $0.000 *$ & & & \\
\hline Radical degree & $1.422(1.083-1.866)$ & $0.011^{*}$ & & & \\
\hline Histological type & $0.889(0.76 \mathrm{I}-1.039)$ & 0.139 & & & \\
\hline TNM stage & $2.804(2.263-3.475)$ & $0.000 *$ & 1.087 & $2.965(2.378-3.698)$ & $0.000 *$ \\
\hline Platelet & $1.001(1.000-1.003)$ & 0.070 & & & \\
\hline Albumin & $0.957(0.939-0.977)$ & $0.000 *$ & & & \\
\hline Fibrinogen & $0.990(0.963-1.018)$ & 0.477 & & & \\
\hline NLR & $1.082(1.023-1.145)$ & $0.006^{*}$ & & & \\
\hline PLR & $1.004(1.002-1.006)$ & $0.000 *$ & & & \\
\hline PNI & $0.958(0.943-0.974)$ & $0.000^{*}$ & -0.028 & $0.972(0.954-0.991)$ & $0.004^{*}$ \\
\hline Adjuvant therapy & $0.928(0.713-1.208)$ & 0.578 & -0.563 & $0.569(0.434-0.747)$ & $0.000 *$ \\
\hline \multicolumn{6}{|c|}{ Model 2 (categorical variables) } \\
\hline Age ( $\leq 60$ vs $>60$ years) & $1.203(0.93 \mid-1.554)$ & 0.158 & & & \\
\hline Gender (male vs female) & $1.350(1.025-1.778)$ & $0.033^{*}$ & & & \\
\hline Diameter $(\leq 4.0$ vs $>4.0)$ & $1.943(1.485-2.543)$ & $0.000 *$ & & & \\
\hline Radical degree (A vs $B)$ & $1.422(1.083-1.866)$ & $0.011 *$ & & & \\
\hline Histological type & $0.889(0.761-1.039)$ & 0.139 & & & \\
\hline \multicolumn{6}{|l|}{ (well vs poorly vs mixed) } \\
\hline TNM stage (I vs II vs III) & $2.804(2.263-3.475)$ & $0.000 *$ & 1.084 & $2.957(2.369-3.690)$ & $0.000 *$ \\
\hline Platelet ( $\leq 300$ vs $>300$ ) & $1.304(0.949-1.791)$ & 0.101 & & & \\
\hline Albumin $(\leq 4.0$ vs $>4.0)$ & $0.512(0.392-0.669)$ & $0.000 *$ & & & \\
\hline Fibrinogen $(\leq 3.5$ vs $>3.5)$ & $1.674(1.287-2.179)$ & $0.000 *$ & & & \\
\hline $\operatorname{NLR}(\leq 3.0$ vs $>3.0)$ & $1.783(1.277-2.49 \mid)$ & $0.00 I^{*}$ & & & \\
\hline $\operatorname{PLR}(\leq 130$ vs $>130)$ & I.447 (I.I I9-I.870) & $0.005^{*}$ & & & \\
\hline $\mathrm{PNI}(\leq 49$ vs $>49)$ & $0.474(0.365-0.615)$ & $0.000 *$ & -0.435 & $0.647(0.497-0.844)$ & $0.001^{*}$ \\
\hline Adjuvant therapy (no vs yes) & $0.928(0.7 \mid 3-1.208)$ & 0.578 & -0.567 & $0.567(0.432-0.745)$ & $0.000 *$ \\
\hline
\end{tabular}

\section{Note: ${ }^{*} p<0.05$.}

Abbreviations: OS, overall survival; GC, gastric cancer; TNM, tumor-node-metastasis; NLR, neutrophil-lymphocyte ratio; PLR, platelet-lymphocyte ratio; PNI, prognostic nutritional index; $\mathrm{HR}$, hazard ratio; $\mathrm{Cl}$, confidence interval.

A

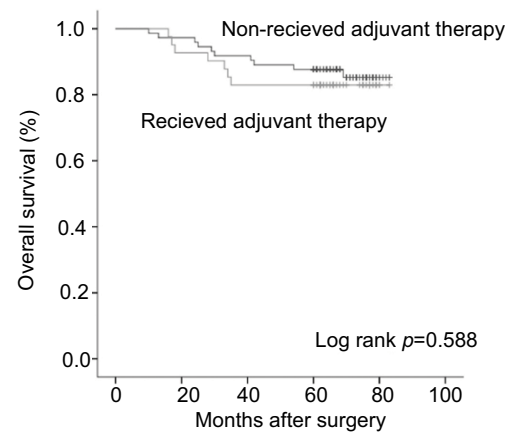

B

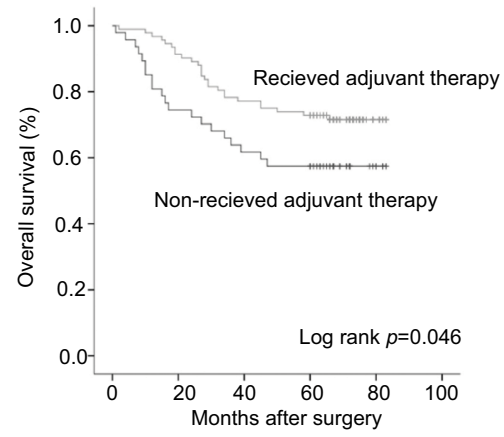

C

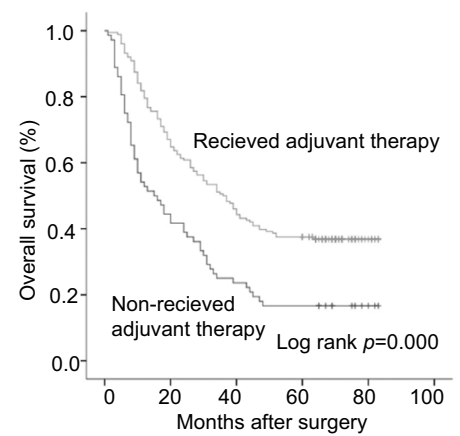

Figure I Postoperative adjuvant therapy improved the OS of stage II and III disease, but not for stage I. (A) Stage I; (B) stage II; (C) stage III. Abbreviation: OS, overall survival.

patients with stage II-III were included as the adjuvant group, while the other 192 patients with stage I-III were included as the non-adjuvant group. The Cox analysis with both models identified the TNM-PNI score as the only inde- pendent prognostic factor of GC, whether adjuvant therapy was administered or not [non-adjuvant: Model 1 and Model 2: HR $(95 \% \mathrm{CI})=1.923(1.656-2.234), p=0.000$; adjuvant: Model 1 and Model 2: HR (95\% CI) $=1.649$ (1.391-1.953), 
A

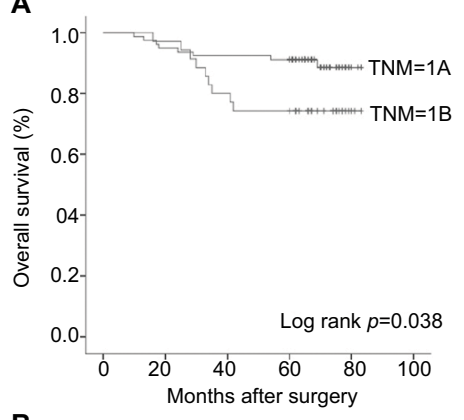

B

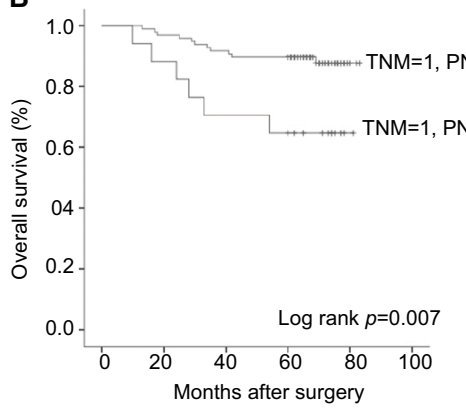

C

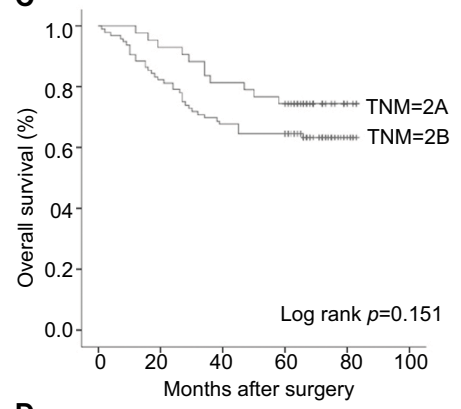

D

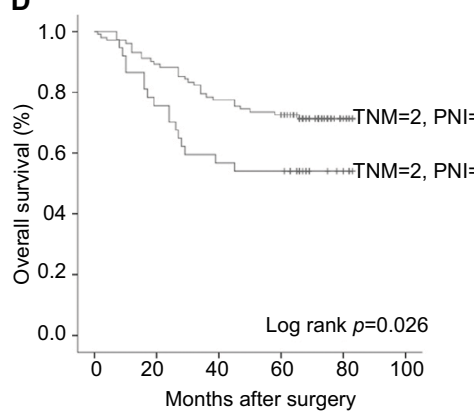

E

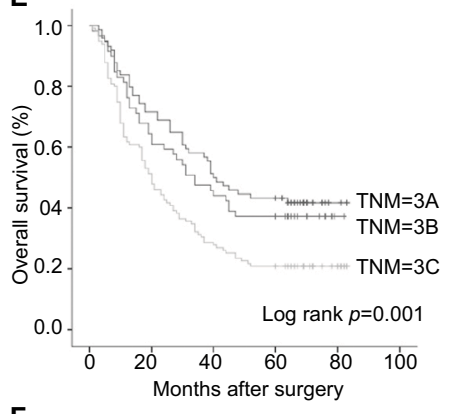

$\mathbf{F}$

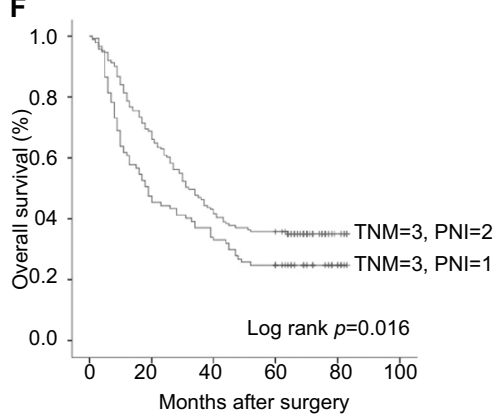

Figure 2 Comparison of OS curves between the dispersed TNM stage and PNI for GC patients with curative D2 resection. Stage I (A vs B); stage II (C vs D); stage III (E vs $\mathbf{F})$.

Abbreviations: OS, overall survival; TNM, tumor-node-metastasis; PNI, prognostic nutritional index; GC, gastric cancer.

A

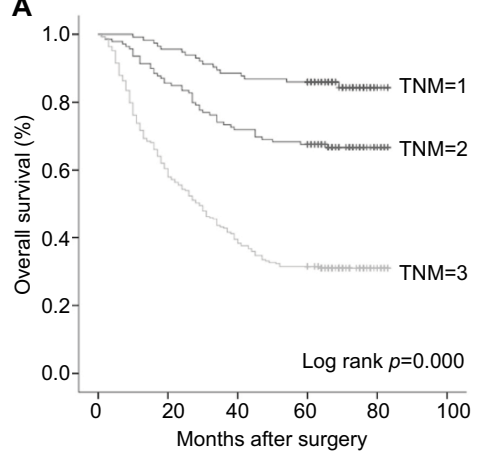

B

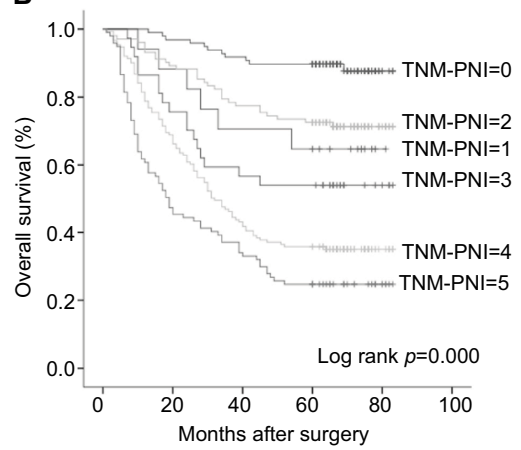

C

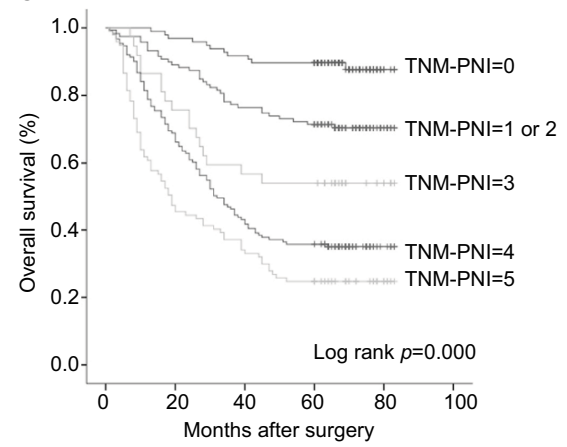

Figure 3 OS curves of TNM stage and the "TNM-PNI" scoring system for GC patients with curative D2 resection. (A) TNM stage; (B) TNM-PNI scoring system; (C) adjusted TNM-PNI scoring system (generalizing TNM-PNI $=\mathrm{I}$ and 2 as one group).

Abbreviations: OS, overall survival; TNM, tumor-node-metastasis; PNI, prognostic nutritional index; GC, gastric cancer.

Table 3 Multivariate survival analysis of OS in GC patients with curative D2 resection according to the NCCN guidelines

\begin{tabular}{lll}
\hline Model I \& Model 2 & HR (95\% Cl) & p-value \\
\hline $\begin{array}{l}\text { Non-received adjuvant therapy } \\
\text { TNM-PNI }\end{array}$ & $1.923(1.656-2.234)$ & $0.000^{*}$ \\
$\begin{array}{l}\text { Received adjuvant therapy } \\
\text { TNM-PNI }\end{array}$ & $1.649(1.391-1.953)$ & $0.000^{*}$ \\
\hline
\end{tabular}

Note: $* p<0.05$.

Abbreviations: OS, overall survival; GC, gastric cancer; TNM, tumor-nodemetastasis; PNI, prognostic nutritional index; NCCN, National Comprehensive Cancer Network; HR, hazard ratio; $\mathrm{Cl}$, confidence interval. $p=0.000]$ (Table 3). As shown in Figure 4A and B, the TNMPNI scoring system could effectively predict the prognosis of GC patients. For patients without adjuvant therapy, there was no significant difference in the prognosis between patients with TNM-PNI $=4$ and 5 (Figure 4A). However, as shown in Table 4, we found that adjuvant therapy might be beneficial for GC patients with TNM-PNI $=4$ or 5 , as they had significantly better long-term survival rates than patients in the non-adjuvant therapy group $(p<0.05)$. 

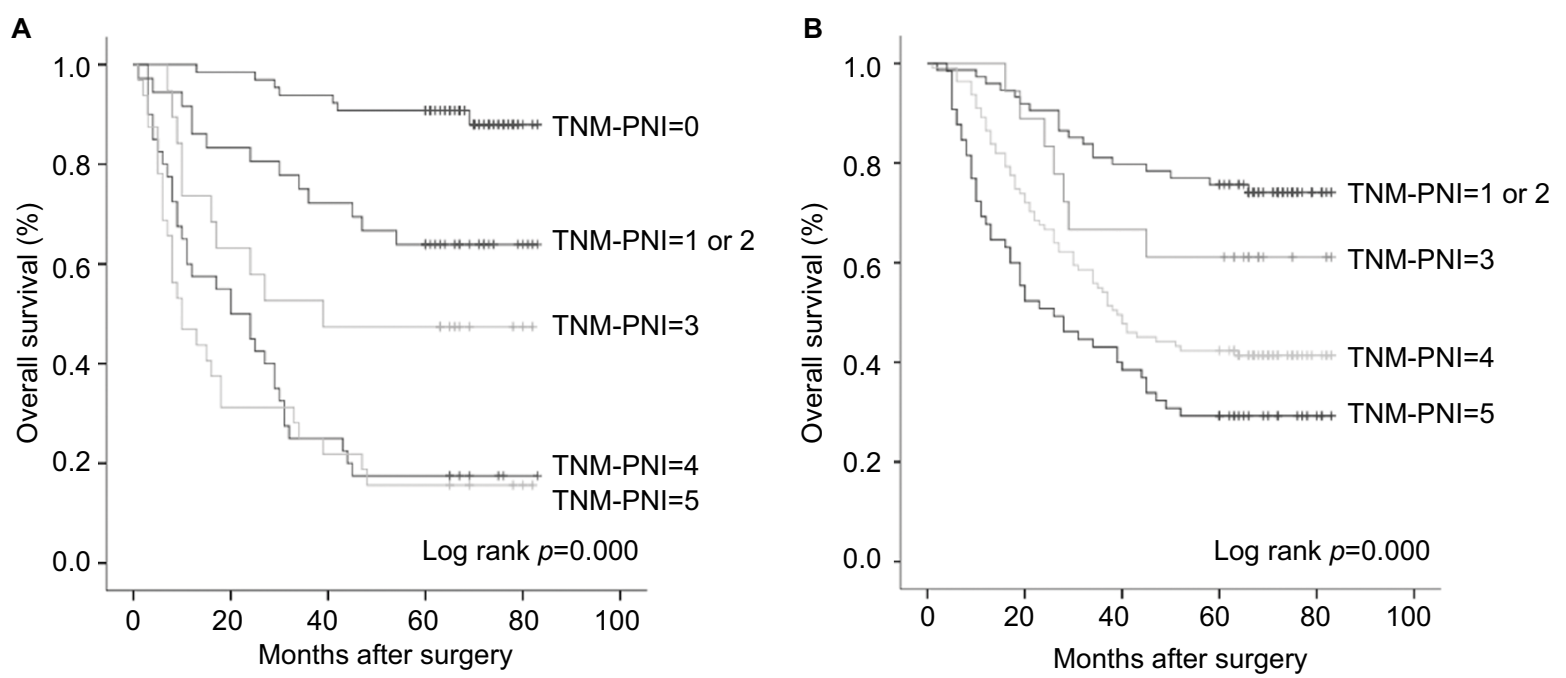

Figure 4 Prognostic value of “TNM-PNI" for GC patients with curative D2 resection according to NCCN guidelines. (A) The survival curve of TNM-PNI in patients without adjuvant therapy; (B) the survival curve of TNM-PNI in patients with adjuvant therapy.

Abbreviations: TNM, tumor-node-metastasis; PNI, prognostic nutritional index; GC, gastric cancer; NCCN, National Comprehensive Cancer Network.

Table 4 The I-, 3-, and 5-year survival rate of the TNM-PNI prognostic score system for GC patients with curative D2 resection according to NCCN guidelines

\begin{tabular}{|c|c|c|c|c|c|c|}
\hline \multirow[t]{2}{*}{ “TNM-PNI" } & \multirow[t]{2}{*}{ Adjuvant therapy } & \multirow[t]{2}{*}{ Number } & \multicolumn{3}{|c|}{ Survival rate (\%) } & \multirow[t]{2}{*}{$\overline{\text { Log-rank } p \text {-value }}$} \\
\hline & & & I-year & 3-year & 5-year & \\
\hline \multirow[t]{2}{*}{0} & No & 65 & 100 & 93.8 & 84.6 & None \\
\hline & Yes & 0 & None & None & None & \\
\hline \multirow[t]{2}{*}{1 or 2} & No & 36 & 86.1 & 72.2 & 55.6 & 0.218 \\
\hline & Yes & 74 & 95.9 & 81.1 & 83.0 & \\
\hline \multirow[t]{2}{*}{3} & No & 19 & 73.7 & 52.6 & 47.4 & 0.242 \\
\hline & Yes & 18 & 100 & 66.6 & 61.1 & \\
\hline \multirow[t]{2}{*}{4} & No & 40 & 57.5 & 25.0 & 17.5 & $0.000 *$ \\
\hline & Yes & $\mathrm{III}$ & 86.5 & 54.1 & $4 I .4$ & \\
\hline \multirow[t]{2}{*}{5} & No & 32 & 46.9 & 25.0 & 15.6 & 0.024 \\
\hline & Yes & 65 & 67.7 & 43.1 & 24.6 & \\
\hline
\end{tabular}

Note: ${ }^{*} p<0.05$.

Abbreviations: OS, overall survival; GC, gastric cancer; TNM, tumor-node-metastasis; PNI, prognostic nutritional index; NCCN, National Comprehensive Cancer Network.

\section{Discussion}

GC is a worldwide problem. Approximately 951,600 new GC cases and 723,100 deaths had occurred because of GC in 2012. ${ }^{1}$ The TNM stage has been widely regarded as the best prognostic factor for cancers, and it has guided clinical work for decades. In our analysis, we have also verified the TNM stage as one of the independent prognostic factors that could effectively reflect the survival time of GC. However, in the clinic, we often found significant survival heterogeneity between GC patients who had the same TNM stage. Thus, in recent years, researchers have changed focus from the tumor itself to the tumor environment, particularly the nutritional and inflammatory status of patients.
Nutritional status always plays an important role in the prognosis of cancer patients, especially among older patients. ${ }^{12}$ For example, albumin, a recognized nutritional index, was one of the independent predictors of OS in GC patients; and its prognostic value has even surpassed that of the TNM stage in some studies. ${ }^{3,13}$ Furthermore, chronic inflammation can also promote and reflect tumor progression by affecting host immunity and the antitumor response. Lymphocytes are crucial components of the immune system for their role in destroying cancer cells, and lymphocyte proliferation and metastasis can be induced by the presence of cancer cells. Tumor-infiltrating lymphocytes are important antitumor immune cells, and their distribution and infiltra- 
tion of tumors are closely related to the oncogenesis and prognosis of tumors. Thus, PNI, a combination of albumin concentration and lymphocyte count, has been considered a good prognostic predictor for cancer patients. In this study, we performed separate Cox multivariate analyses for continuous and categorical variables and demonstrated that TNM stage, PNI, and adjuvant therapy were independent prognostic factors of $\mathrm{GC}$, consistent with previous reports.

In addition to other inflammatory markers, we also found PNI to be associated with tumor size and TNM stage (Table S1). Tumor size is a manifestation of tumor burden and a prognostic factor for several tumors. GC patients with earlier TNM stage always have better nutritional and immune status. Thus, PNI, a reflection of physical condition, is always negatively correlated with tumor burden and tumor stage. We realized that PNI could be a prognostic factor in addition to the TNM stage. As shown in Figure 2, PNI could separate the survival curve more effectively than the TNM stage, especially for stage I and II GC patients. Other biochemical markers may also be associated with the prognosis of cancers. Several studies have reported that platelets contribute to the multistep development of tumors and could be a therapeutic target for tumors. ${ }^{14-16}$ Fibrinogen is a pro-inflammatory protein that plays an important role in inflammatory responses and tumor progression; it is associated with the clinicopathologic features and prognosis of several tumors, such as breast, lung, prostate, and gastrointestinal cancers. ${ }^{17-21}$ In addition, some meta-analysis demonstrated that NLR and PLR could also predict the prognosis for several tumors. However, in our analysis, the biomarkers mentioned above were not independent prognostic factors for GC patients; all of them were associated with the preoperative PNI. Overall, we consider PNI to be a better prognostic biomarker for GC.

In this study, we established the TNM-PNI scoring system according to the results of multivariate analysis. We also demonstrated that it was a highly accurate and practical system for evaluating the prognosis of GC, because it reflects the tumor stage, inflammation status, and nutritional status of every patient. For GC, a higher TNM-PNI score has always indicated a worse cancer outcome. We verified that TNM-PNI was the major independent prognostic factor for GC patients with curative $\mathrm{D} 2$ resection, independent of whether patients received or did not receive adjuvant therapy. In addition, we recommend adjuvant therapy for stage II and III GC, but not for stage I, which is consistent with the NCCN guidelines. Furthermore, we have found that the adjuvant therapy might be more beneficial for the survival of $\mathrm{GC}$ patients with TNM-PNI $=4$ and 5 , and it plays an important role in postoperative therapy for GC. Thus, our study revealed that TNM-PNI may be a good supplement for the NCCN guidelines for GC and could provide guidance for clinical work.

As far as we know, this is the first report of TNM-PNI as a novel scoring system. However, there are also some limitations to the present study. First, due to the retrospective nature of our research, additional potential prognostic indexes, such as C-reactive protein, CD4/CD8 lymphocyte count, and modified GPS could not be obtained and included in our analysis. Second, clinicopathologic data could not be obtained for some of the patients, who were, therefore, excluded from the analysis. Consequently, the sample size of this study was relatively small. Finally, our results are based on the TNM stage according to the 7th edition of the AJCC system, and it remains to be seen whether our results can be replicated for other staging systems.

\section{Conclusion}

TNM stage, preoperative PNI, and adjuvant therapy were closely related to the prognosis of GC patients. The PNI had a distinctly subsidiary role relative to the TNM stage in predicting patient survival time. Combining both factors, we established the novel TNM-PNI scoring system and demonstrated that patients with a higher TNM-PNI score always had worse survival outcomes. In conclusion, the TNM-PNI score is a highly effective prognostic factor for GC patients with curative D2 resection, and it can be a good supplement for the NCCN guidelines.

\section{Acknowledgments}

This study was funded by the Natural Science Foundation of Liaoning Province (No. 2015020500). The funders had no role in the study design, data collection and analysis, and preparation or publication of the manuscript.

\section{Disclosure}

The authors report no conflicts of interest in this work.

\section{References}

1. Torre LA, Bray F, Siegel RL, Ferlay J, Lortet-Tieulent J, Jemal A. Global cancer statistics, 2012. CA Cancer J Clin. 2015;65(2):87-108.

2. Zitvogel L, Pietrocola F, Kroemer G. Nutrition, inflammation and cancer. Nat Immunol. 2017;18(8):843-850.

3. Liu BZ, Tao L, Chen YZ, et al. Preoperative body mass index, blood albumin and triglycerides predict survival for patients with gastric cancer. PLoS One. 2016;11(6): e0157401.

4. Hirashima K, Watanabe M, Shigaki H, et al. Prognostic significance of the modified Glasgow prognostic score in elderly patients with gastric cancer. J Gastroenterol. 2014;49(6):1040-1046. 
5. Jiang $X$, Hiki N, Nunobe S, et al. Prognostic importance of the inflammation-based Glasgow prognostic score in patients with gastric cancer. Br J Cancer. 2012;107(2):275-279.

6. Graziosi L, Marino E, De Angelis V, Rebonato A, Cavazzoni E, Donini A. Prognostic value of preoperative neutrophils to lymphocytes ratio in patients resected for gastric cancer. Am J Surg. 2015;209(2):333-337.

7. Ock CY, Nam AR, Lee J, et al. Prognostic implication of antitumor immunity measured by the neutrophil-lymphocyte ratio and serum cytokines and angiogenic factors in gastric cancer. Gastric Cancer. 2017;20(2):254-262.

8. Shimada H, Takiguchi N, Kainuma O, et al. High preoperative neutrophil-lymphocyte ratio predicts poor survival in patients with gastric cancer. Gastric Cancer. 2010;13(3):170-176.

9. Migita K, Takayama T, Saeki K, et al. The prognostic nutritional index predicts long-term outcomes of gastric cancer patients independent of tumor stage. Ann Surg Oncol. 2013;20(8):2647-2654.

10. Sun K, Chen S, Xu J, Li G, He Y. The prognostic significance of the prognostic nutritional index in cancer: a systematic review and metaanalysis. J Cancer Res Clin Oncol. 2014;140(9):1537-1549.

11. Sun KY, Xu JB, Chen SL, et al. Novel immunological and nutritionalbased prognostic index for gastric cancer. World J Gastroenterol. 2015;21(19):5961-5971.

12. Liu X, Qiu H, Kong P, Zhou Z, Sun X. Gastric cancer, nutritional status, and outcome. Onco Targets Ther. 2017;10:2107-2114.

13. Sun H, He B, Nie Z, A nomogram based on serum bilirubin and albumin levels predicts survival in gastric cancer patients. Oncotarget. 2017;8(25):41305-41318.
14. Xie X, Zeng X, Cao S, et al. Elevated pretreatment platelet distribution width and platelet count predict poor prognosis in nasopharyngeal carcinoma. Oncotarget. 2017;8(62):106089-106097.

15. Franco AT, Corken A, Ware J. Platelets at the interface of thrombosis, inflammation, and cancer. Blood. 2015;126(5):582-588.

16. Rachidi S, Wallace K, Day TA, Alberg AJ, Li Z. Lower circulating platelet counts and antiplatelet therapy independently predict better outcomes in patients with head and neck squamous cell carcinoma. $J$ Hematol Oncol. 2014;7:65.

17. Zhang J, Li SQ, Liao ZH, et al. Prognostic value of a novel FPR biomarker in patients with surgical stage II and III gastric cancer. Oncotarget. 2017;8(43):75195-75205.

18. Wang $\mathrm{Y}, \mathrm{Chen} \mathrm{W}, \mathrm{Hu} \mathrm{C}$, et al. Albumin and fibrinogen combined prognostic grade predicts prognosis of patients with prostate cancer. $J$ Cancer. 2017;8(19):3992-4001.

19. Wang H, Zhao J, Zhang M, Han L, Wang M, Xingde L. The combination of plasma fibrinogen and neutrophil lymphocyte ratio (F-NLR) is a predictive factor in patients with resectable non small cell lung cancer. J Cell Physiol. 2018;233(5):4216-4224.

20. Liu FT, Gao H, Wu CW, Zhu ZM. The association of plasma fibrinogen with clinicopathological features and prognosis in esophageal cancer patients. Oncotarget. 2017;8(54):93029-93038.

21. Fan S, Guan Y, Zhao G, An G. Association between plasma fibrinogen and survival in patients with small-cell lung carcinoma. Thorac Cancer. 2018;9(1):146-151. 


\section{Supplementary materials}

Table SI Association between preoperative $\mathrm{PNI}$ and clinicopathologic features of 50I GC patients with curative D2 resection

\begin{tabular}{|c|c|c|c|}
\hline Variables & $P N I=I(n=\mid 5 I)$ & $P N I=2(n=350)$ & $p$ value \\
\hline Age, years & $61.17 \pm 11.16$ & $58.39 \pm 10.62$ & $0.002 *$ \\
\hline Gender & & & 0.093 \\
\hline Male & 102 & 262 & \\
\hline Female & 49 & 88 & \\
\hline Diameter & $5.82 \pm 2.75$ & $4.36 \pm 2.01$ & $0.000 *$ \\
\hline Radical degree & & & 0.105 \\
\hline A & 101 & 259 & \\
\hline B & 50 & 91 & \\
\hline Histological type & & & 0.957 \\
\hline Well differentiated & 34 & 78 & \\
\hline Poorly differentiated & 77 & 178 & \\
\hline Mixed & 40 & 94 & \\
\hline TNM stage & & & $0.000 *$ \\
\hline 1 & 17 & 97 & \\
\hline ॥ & 37 & 102 & \\
\hline III & 97 & $15 \mid$ & \\
\hline T stage & & & $0.000 *$ \\
\hline $\mathrm{TI}$ & 8 & 81 & \\
\hline $\mathrm{T} 2$ & 22 & 56 & \\
\hline T3 & 16 & 47 & \\
\hline $\mathrm{T} 4$ & 105 & 166 & \\
\hline N stage & & & $0.000 *$ \\
\hline No & 37 & 152 & \\
\hline NI & 23 & 66 & \\
\hline N2 & 28 & 59 & \\
\hline N3 & 63 & 73 & \\
\hline Platelet & $242.30 \pm 82.47$ & $231.30 \pm 81.86$ & 0.110 \\
\hline Albumin & $36.75 \pm 5.00$ & $44.22 \pm 3.85$ & $0.000 *$ \\
\hline Fibrinogen & $4.96 \pm 9.60$ & $3.65 \pm 1.05$ & $0.002 *$ \\
\hline NLR & $2.99 \pm 2.39$ & $1.80 \pm 0.76$ & $0.000 *$ \\
\hline PLR & $176.82 \pm 8 \mid .32$ & || $6.74 \pm 5 \mid .57$ & $0.000 *$ \\
\hline Adjuvant therapy & & & 0.821 \\
\hline No & 59 & 133 & \\
\hline Yes & 92 & 217 & \\
\hline
\end{tabular}

Note: ${ }^{*} p<0.05$.

Abbreviations: GC, gastric cancer; TNM, tumor-node-metastasis; NLR, neutrophil-lymphocyte ratio; PLR, platelet-lymphocyte ratio; PNI, prognostic nutritional index.
Table S2 Multivariate survival analysis of OS in 50 I GC patients with curative D2 resection after establishing TNM-PNI

\begin{tabular}{lll}
\hline Model I \& Model 2 & HR $(95 \%$ Cl) & -value \\
\hline TNM-PNI & I.8I2 $(I .632-2.0 I 0)$ & $0.000^{*}$ \\
Adjuvant therapy & $0.597(0.456-0.782)$ & $0.000^{*}$ \\
\hline
\end{tabular}

Note: $* p<0.05$.

Abbreviations: OS, overall survival; GC, gastric cancer; TNM, tumor-nodemetastasis; $\mathrm{PNI}$, prognostic nutritional index; $\mathrm{HR}$, hazard ratio; $\mathrm{Cl}$, confidence interval.
Cancer Management and Research

\section{Publish your work in this journal}

Cancer Management and Research is an international, peer-reviewed open access journal focusing on cancer research and the optimal use of preventative and integrated treatment interventions to achieve improved outcomes, enhanced survival and quality of life for the cancer patient. The manuscript management system is completely online and includes

\section{Dovepress}

a very quick and fair peer-review system, which is all easy to use. Visit http://www.dovepress.com/testimonials.php to read real quotes from published authors. 